\title{
ANÁLISIS DEL GRADO DE SATISFACCIÓN DEL PROFESORADO DE EDUCACIÓN SECUNDARIA EN EL DESARROLLO DE SU LABOR DOCENTE*
}

\author{
José Antonio Torres González y \\ Equipo de Investigación Grupo D.I.E.A.** \\ Universidad de Jaén
}

\begin{abstract}
RESUMEN: Este artículo es fruto de un proyecto de investigación en el que se trata de indagar sobre el grado de satisfacción de los profesionales de Educación Secundaria en el desempeño de su labor docente. Se analizan, de manera general, distintas dimensiones relacionadas con los niveles de satisfacción-insatisfacción en el ejercicio profesional, con las relaciones personales, con las estructuras organizativas de los centros y con la estructura y las bases teóricas que fundamentan el desarrollo normativo de la Educación Secundaria en el sistema educativo actual. Igualmente se aborda, de manera más específica, lo que "dicen hacer" respecto a aspectos curriculares referidos a contenidos, metodología y evaluación. A partir del análisis de las respuestas del cuestionario elaborado se plantean una serie de propuestas que intentan generar procesos de mejora en el desarrollo profesional de este colectivo de docentes.
\end{abstract}

ABSTRACT: This article is fruit of a project of investigation in which is a matter of investigating on the degree of satisfaction of the professionals of Secondary Education in the performance of its educational work. They are analyzed, in a general way, different dimensions related to the levels of satisfactiondissatisfaction, in the professional exercise, in the personal relations, with the organizing structures of the centers and with the structure and the theoretical

* Este trabajo es producción científica derivada del Proyecto I+D financiado por el Ministerio de Ciencia y Tecnología (Orden de 31 de Enero de 2001, BOE del 2 de Febrero, Ref. BSO2001-3800).

** Equipo de Investigación Grupo DIEA: María Jesús Colmenero Ruiz, Lorenzo Almazán More-

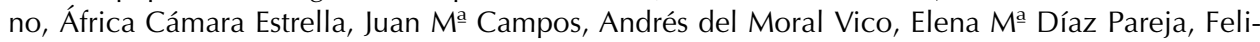
pe García Mino, Ma Dolores Hueso Villegas, David Molero López-Barajas, Juana Ma Ortega Tudela, Ana Ortiz Colón, Maa Ángeles Peña Hita, Manuel Román Rayo, Encarnación Rueda, Juan Ruiz Carrascosa. 
bases that support the normative development of the Secondary Education in the present educational system. Subsequently it is undertaken, of more specific way, what "they say to do" with respect to curriculum aspects referred to contents, methodology and evaluation. From the analysis of the answers of the elaborate questionnaire they are presented a series of proposals that would seek to improve the professional development of this collective one of professionals.

PALABRAS CLAVE: Satisfacción, insatisfacción, formación, motivación, docencia, currículum, evaluación.

KEYWORDS: Satisfaction, dissatisfaction, formation, motivation, teaching, curriculum, evaluation.

\section{INTRODUCCIÓN}

Son múltiples los problemas de una sociedad cambiante y plural. Éstos son diagnosticados señalando ineludiblemente a la institución educativa como espacio público, como cruce de fronteras capacitada para resolverlos o prevenirlos antes de que sean más difíciles de erradicar. Ello demanda perfiles de profesionales con unas exigencias de formación más especializadas que generalistas, presionados para desarrollar una amplísima gama de competencias en un contexto laboral cambiante e inestable. El cambio de los diferentes sistemas educativos que se vienen implantando les obliga a permanecer en un estado de continuo reciclaje en el proceso de enseñanza-aprendizaje en los términos del aprendizaje significativo y del "aprender a aprender". Como contrapartida, no se les ofrece un reconocimiento profesional y un apoyo social a su tarea. Pero es obvio que en los sistemas educativos, el éxito de la implantación del actual sistema sin ir más lejos, se basa en gran parte directamente en el profesorado, por lo que nos planteamos en nuestro estudio explorar el grado de satisfacción-insatisfacción profesional percibido por este colectivo como fundamento de la motivación para el buen desempeño de su tarea docente y formadora. Somos conscientes de que esta realidad, si bien ha sido objeto de estudio junto con otros aspectos del sistema educativo, es ahora cuando comienza a percibirse como una preocupación creciente en este ámbito. Se comprende así el interés por investigar el problema, no sólo por parte de los expertos universitarios, sino también por parte de otros colectivos sociales.

Nuestra investigación se centra en la etapa de la Educación Secundaria Obligatoria, en distintos aspectos cuya resolución definiría las claves del bienestar del profesorado: problemas de formación, de desarrollo de los procesos de enseñanza-aprendizaje, de pautas de colaboración en el desempeño de su labor docente, de relaciones con los alumnos y colegas y de nuevas situaciones en el aula, en referencia a las estructuras organizativas de los centros educativos. Pensamos que todo esfuerzo por mejorar la calidad docente y, por extensión, educativa, pasaría, en parte, por solucionar las carencias que en tales ámbitos se nos plantean.

\section{Estado de la CUESTIÓN SObRe SATISFACCión Del PROFESORADO}

La satisfacción profesional de los docentes puede entenderse como el resultado de comparar lo que la realidad le invita a ser y lo que según sus expectativas debe- 
ría ser. Marcelo (1995), desde la perspectiva de la satisfacción, entendida en el marco del desarrollo profesional, incide, entre otros factores aclaratorios, sobre el desarrollo profesional, que debe estar basado en un proceso de mejora profesional, donde se tengan en cuenta las necesidades prácticas y contextualizadas de los docentes. Consideramos que la satisfacción laboral es uno de los indicadores más clásicos a los que se suele recurrir, cuando se intenta conocer cual es la actitud general de los sujetos hacia su vida profesional (Peiró y cols., 1991). No podemos olvidar que la tarea docente-investigadora amén de profesión es vocación.

En la revisión de investigaciones sobre la satisfacción del profesorado en la realización de la docencia en la Educación Obligatoria, queremos destacar algunas de ellas por la relevancia de sus aportaciones. En este sentido, Pérez (1997) llevó a cabo una investigación sobre las condiciones necesarias para el desarrollo profesional del profesorado, y subraya entre otras causas, que una de las situaciones por la que atraviesa la profesión docente es que presenta desajustes entre exigencias y recompensas, donde se genera un conflicto por la falta de concordancia entre la idealización profesional y las condiciones reales de su ejercicio. Desde la perspectiva de las condiciones de trabajo, Conley y cols. (1989), ya consideraban que las condiciones de trabajo influyen de manera directa sobre la profesión docente. Enumeran una serie de factores para evaluar las condiciones de trabajo de los docentes. Estos elementos son el tipo de tarea, cargas y esfuerzos, concentración sobre la tarea, horario laboral, ambiente en el trabajo, aspectos socioeconómicos, características individuales del trabajador y lugar de trabajo. Se han realizado recientemente en nuestro país diferentes investigaciones sobre necesidades del profesorado de Educación Secundaria Obligatoria que hacen referencia a las fuentes de satisfacción e insatisfacción con su tarea como educadores. En ellas se ponen de manifiesto cuáles son, desde el punto de vista de los docentes, las facetas más decisivas de su trabajo, las cuestiones que para el profesorado resultan ser cruciales para llevar a cabo su tarea de manera satisfactoria. En este sentido, los resultados ofrecidos en el último informe del INCE (2003) referidos a los profesores de ESO de centros de titularidad pública, así como los del estudio de Marchesi y Martín (2002), son bastante ilustrativos. Se refleja que para la mayor parte de los docentes de ESO, las principales fuentes de satisfacción en el desarrollo de su labor docente proceden de la relación que mantienen tanto con sus alumnos como con sus compañeros, incluyendo al equipo directivo. Similares resultados se han obtenido en el estudio de Barquín (2002, cit. en Pérez, Sola, Blanco y Barquín, 2003), realizado con una amplia muestra de profesores andaluces de ESO. Aunque por el contrario, teniendo en cuenta la "Encuesta de opinión del profesorado sobre la calidad de la educación" (Martín, Pérez y Álvarez, 2007) elaborada por la Fundación Hogar del Empleado y efectuada por el Instituto de Evaluación y Asesoramiento Educativo (IDEA), vemos que la gran mayoría de los profesores no se sienten valorados por la sociedad ni por la administración por lo que ese sentimiento puede llegar a convertirse en una traba, si bien la gran mayoría (82\%) de los mismos no cambiaría su profesión. Por otra parte, Sanmartín (2008) ${ }^{1}$ asegura que el profesorado de Educación Secundaria está muy capacitado para la docencia de sus materias

1. http://www.soitu.es/soitu/2008/03/27/info/1206634348_499177.html. 
pero nadie les forma en la resolución de conflictos. Este hecho queda reflejado en centros conflictivos en los que el profesorado se siente indefenso, situación que conIleva una insatisfacción laboral, ya que no pueden desempeñar su tarea adecuadamente, así como disfrutar de su profesión.

Siguiendo en la búsqueda de trabajos preliminares sobre el tema, que nos sitúen en el estado de la cuestión, hemos analizado diferentes tesis doctorales que han tratado las condiciones laborales del docente (www.mcu.es/TESEO/):

La tesis de Vera (1986) "Incidencias de los factores socio-laborales en el profesor debutante" profundiza sobre las fuentes de malestar y de satisfacción más relevantes que afectan al profesorado de enseñanzas básicas de Málaga y provincia cuando se encuentra en los primeros 5 años de ejercicio profesional.

En el trabajo de Ortiz (1993), "El malestar del docente: la enseñanza media como unidad de referencia empírica" se valora la incidencia del malestar docente en el profesorado de EE.MM. de la ciudad y provincia de Salamanca, en términos de presencia de ansiedad, cansancio y depreciación del yo profesional, que afecta al $44 \%$ del profesorado analizado. La investigación desarrolla diferentes propuestas de evitación y ayuda ante los problemas mencionados, enfocadas en la promoción de habilidades relacionales en el profesorado, como condición esencial para impulsar su satisfacción profesional y mejorar la práctica docente. El estudio de Padrón (1994) "Satisfacción profesional del profesorado" Ilevado a cabo en Tenerife, con una muestra de 637 trabajadores (435 profesores y 202 no docentes de diferentes profesiones) es bastante significativo. En toda la muestra la satisfacción profesional es moderada, 2/3, mostrándose $1 / 4$ muy insatisfecho. Los objetivos fundamentales de esta investigación fueron: conocer el nivel de insatisfacción profesional del profesorado que ejerce en la enseñanza no universitaria, en cada una de las dimensiones de su actividad docente, y comparar el nivel de insatisfacción profesional del profesorado de la enseñanza no universitaria con el nivel de insatisfacción profesional de otros trabajadores no docentes y que, por su nivel de cualificación, podrían ejercer también la profesión de docente.

Por otra parte, destacamos distintas investigaciones que giran en torno a las condiciones laborales de los docentes y entre las que subrayamos los estudios de los siguientes autores:

Veenman (1988) realiza un trabajo donde revisa una serie de estudios sobre problemas percibidos por los profesores, entre los que destaca la motivación del alumnado, relación con los padres y colegas de la profesión, carga docente e insuficiencia de material.

Gordillo (1988) describe la satisfacción/insatisfacción de los profesores, no sólo de un modo cuantitativo sino también cualitativo.

Marcelo (1992) ahonda sobre los problemas del profesorado, entre los que enfatiza la inestabilidad laboral de los interinos, la carga docente, la masificación del alumnado y la insuficiente dotación de infraestructura y de materiales didácticos de los centros educativos.

González Blasco y González Anleo (1993) indican que el profesorado muestra su insatisfacción por aspectos relacionados con su puesto de trabajo desde la sociología del mismo, actuación docente y orientación psicológica y psicopatológica. Estos autores consideran que el $80 \%$ del profesorado español muestra insatisfacción laboral. 
La investigación de Sevilla y Villanueva (2000) pretende conocer los principales elementos de insatisfacción del profesorado de la enseñanza pública no universitaria respecto a sus condiciones profesionales y laborales, así como posibles alternativas de solución, todo ello desde su propia percepción, y averiguando aspectos concretos de su realidad laboral.

Marchesi (1990, 2003); Evans (2000); Dinham y Scott (2000) y Bogler (2001) señalan varios factores que influyen en las condiciones laborales y satisfacción del profesorado, como los incentivos económicos, la formación, la promoción profesional y el reconocimiento social.

\section{Metodología}

\subsection{Propósito general y objetivos del estudio}

El propósito general de la investigación es indagar acerca de los niveles de satisfacción del profesorado en el ejercicio de su labor docente. De este propósito general se desprenden los siguientes objetivos:

- Detectar el nivel de satisfacción-insatisfacción en el desarrollo de su ejercicio profesional.

- Analizar el grado de satisfacción-insatisfacción en las relaciones personales con colegas, con alumnos, con familias y con la administración-supervisión.

- Conocer si la estructura organizativa del centro genera satisfacción-insatisfacción en relación con las dimensiones anteriores.

- Detectar si le ayuda en su trabajo la estructura de la Educación Secundaria en el sistema educativo actual.

- Averiguar el grado de satisfacción-insatisfacción con las bases teóricas que fundamentan el desarrollo normativo y organizativo de la Educación Secundaria.

En esta investigación se emplea como instrumento el cuestionario. La orientación predominante tiene en principio un carácter descriptivo y posteriormente un carácter iluminativo, en la medida en que el propósito general que se persigue es describir y tratar de iluminar la evolución del nivel de satisfacción de los profesionales en los centros de la Comunidad Autónoma de Andalucía, así como el sentido, significado y reconstrucción personal e institucional que supone para el profesorado que cotidianamente participa en el proceso. Por todo ello optamos por un modelo de investigación descriptivo con la finalidad de relatar la estructura de los fenómenos y su dinámica, a la vez que identificar aspectos relevantes de la realidad. Ello nos ha permitido acceder al campo con una visión amplia que se cierra con la aplicación de un instrumento como el cuestionario y el análisis posterior de los datos, en la medida en que mejor se pueden lograr los objetivos de investigación.

\subsection{Muestra}

El sistema de muestreo se realiza mediante conglomerados. De las ocho capitales de provincia que componen la Comunidad Autónoma de Andalucía, espacio-campo donde se lleva a cabo la investigación, se seleccionan al azar cuatro centros de cada una. Un total de 32 centros y 902 profesores constituyen la muestra de este estudio. 


\subsection{Instrumentos de recogida de información}

Desde el punto de vista de la investigación social, "las preguntas de un cuestionario son la expresión, en forma interrogativa de las variables empíricas, o indicadores respecto de los cuales interesa obtener información". (González Río, 1997). En cualquier trabajo de investigación o de evaluación es necesario hacer operativas las variables sobre las que vamos a recabar información. Cuando trabajamos con variables en un proceso de investigación descriptiva, o de evaluación, solemos operativizarla en dimensiones. El cuestionario elaborado como instrumento de recogida de información consta de 175 ítems configurados en torno a seis dimensiones:

- Nivel de satisfacción-insatisfacción en la docencia.

- Nivel de satisfacción-insatisfacción en el desarrollo de su ejercicio profesional.

- Grado de satisfacción-insatisfacción en las relaciones personales con colegas, con alumnos, con familias y con la administración-supervisión.

- Nivel de satisfacción-insatisfacción con la estructura organizativa de los centros.

- Nivel de satisfacción-insatisfacción con la estructura de la Educación Secundaria en el sistema educativo actual.

- Grado de satisfacción-insatisfacción con las bases teóricas que fundamentan el desarrollo normativo y organizativo de la Educación Secundaria.

Consideramos que la variable dependiente (nivel de insatisfacción-satisfacción) puede explicarse a partir de de otros factores o variables independientes (docencia, ejercicio profesional, relaciones personales, estructura de los centros, estructura de la Educación Secundaria Obligatoria y bases teóricas que fundamentan el desarroIlo normativo y organizativo de la misma)

La estructura del cuestionario está configurada por un número determinado de ítems de cada dimensión, así como un último ítem en el que se pedía la opinión del profesorado sobre la dimensión en general, que es el objeto de estudio de este artículo.

Para corroborar de forma estadística la validez de nuestro instrumento realizamos un análisis de conglomerados jerárquicos centrado en las variables, utilizando como medida de distancia entre las frecuencias de Pearson y con un rango de soluciones apropiado (hasta 10 o 12 conglomerados) que permitiera obtener alguna que de manera nítida podamos asimilar a la solución. Al alcanzar doce conglomerados se dibuja de manera muy clara una estructura, sin más que adjudicarlos según los coeficientes, en la que los mismos se agrupan en estructuras totalmente similares a la del cuestionario

El cálculo de la fiabilidad se ha realizado mediante el alfa de Cronbach. A través de los resultados obtenidos podemos comprobar que todos los ítems del cuestionario han obtenido coeficientes de fiabilidad superiores a 0,90. Por consiguiente podemos decir que presentan semejanza conceptual y que son suficientemente homogéneos y satisfactorios. 


\section{Resultados De la inVESTigación}

\section{Dimensión 1. Nivel de insatisfacción-satisfacción en la docencia}

En general, con la docencia me siento:

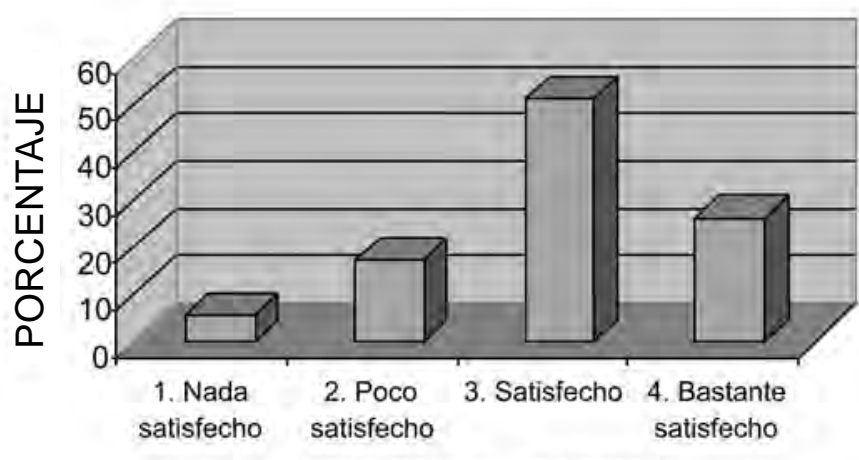

NIVEL DE SATISFACCIÓN

Gráfico 1. Dimensión 1.

Se puede observar que la gran mayoría de los profesores encuentran satisfactorio su trabajo docente $(51 \%)$, siendo muy aproximados los valores que se sitúan en los extremos: (Poco satisfactorio 22,6\% o bastante satisfactorio 26,1\%). Es interesante destacar el valor extremadamente bajo que da el ítem "nada satisfactorio" (5,5\%), entendiéndose, sin lugar a dudas, que la percepción que tiene el profesorado sobre su trabajo siempre tiene "algo" de satisfactorio. Puede afirmarse que los profesionales encuentran un buen nivel de satisfacción con la docencia que imparten.

\section{Dimensión 2. Nivel insatisfacción-satisfacción en el desarrollo de su ejercicio profesional}

En general, con el ejercicio de mi profesión me siento:

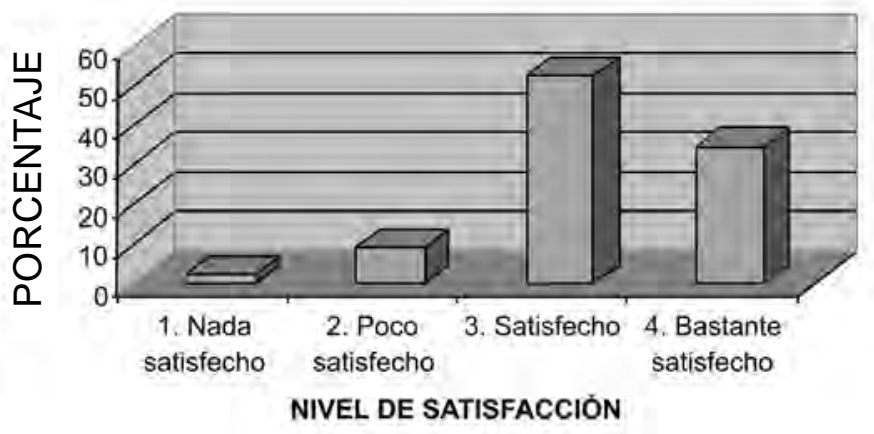

Gráfico 2. Dimensión 2. 
En general, el 88,30\% se siente satisfecho (y bastante satisfecho) con el desarroIlo de su ejercicio profesional que incluye aspectos relacionados con los elementos básicos del currículum y fundamentalmente con su proceso de formación permanente, en el sentido de que ésta le ayuda a resover problemas en la práctica cotidiana del aula. La distancia en tanto por ciento entre "nada" y "poco" satisfecho es de 7 puntos lo cual resulta muy significativo.

\section{Dimensión 3. Nivel de insatisfacción-satisfacción en las relaciones con la supervisión educativa, con la familia, con los colegas, con alumnos y con otros centros educativos próximos del entorno}

En general, en las relaciones con la Supervisión Educativa, me siento:

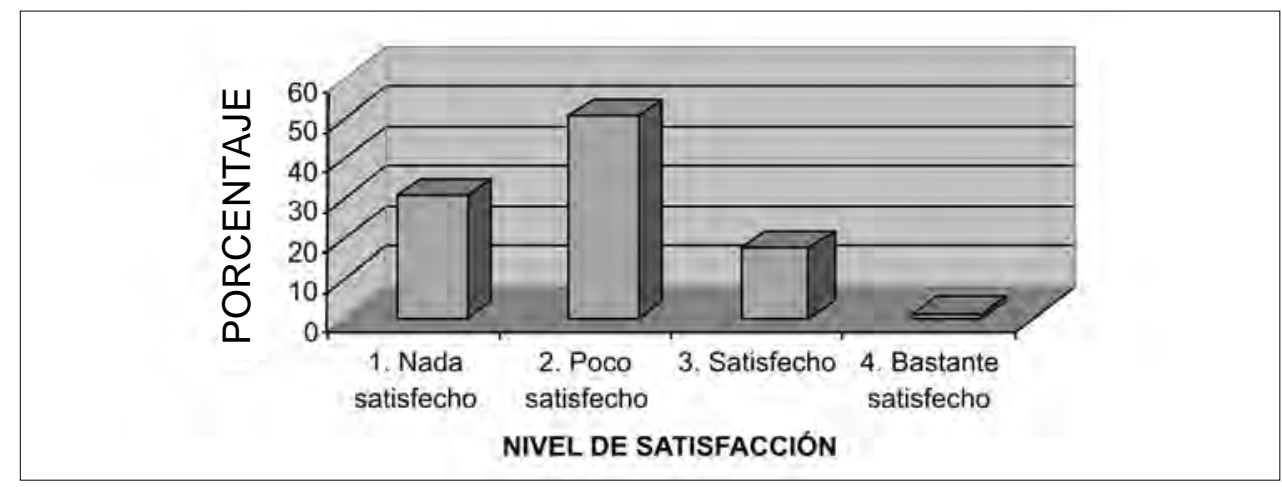

Gráfico 3. Dimensión 3.

En el gráfico podemos apreciar un alto grado de insatisfacción en las relaciones con la administración y en concreto con los responsables de la supervisión educativa (el 81,1\% se muestra poco a nada satisfecho). La visión que los docentes tienen sobre las personas que gestionan administrativamente su trabajo no es muy halagüeña, resulta sorprendente ya que en realidad el contacto entre ambos sectores es puntual y distanciado en el tiempo.

En general, en las relaciones de la familia con el centro, me siento:

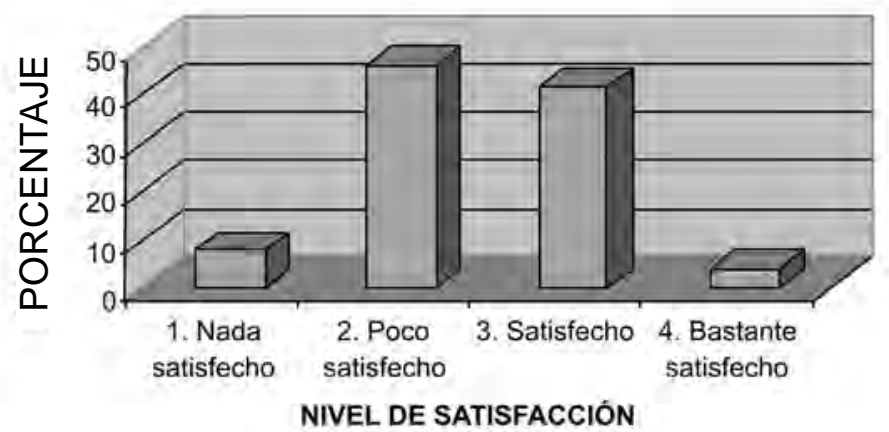

Gráfico 4. Dimensión 3. 
Más de la mitad del profesorado se encuentra poco a nada satisfecho en el proceso relacional entre padres y profesores $(52,2 \%)$. Tan sólo un $4 \%$ se muestra bastante satisfecho en las relaciones de las familias con el centro educativo y por ende con el profesorado. Ciertamente al profesional de la educación secundaria no se le prepara en el ámbito de la interacción que se produce en las tutorías con las familias. Es un aspecto muy delicado y que salvo que se tenga la formación adecuada y experiencia oportuna suele producir roces y malentendidos entre padres y profesorado.

En general, en las relaciones con mis compañeros, me siento:

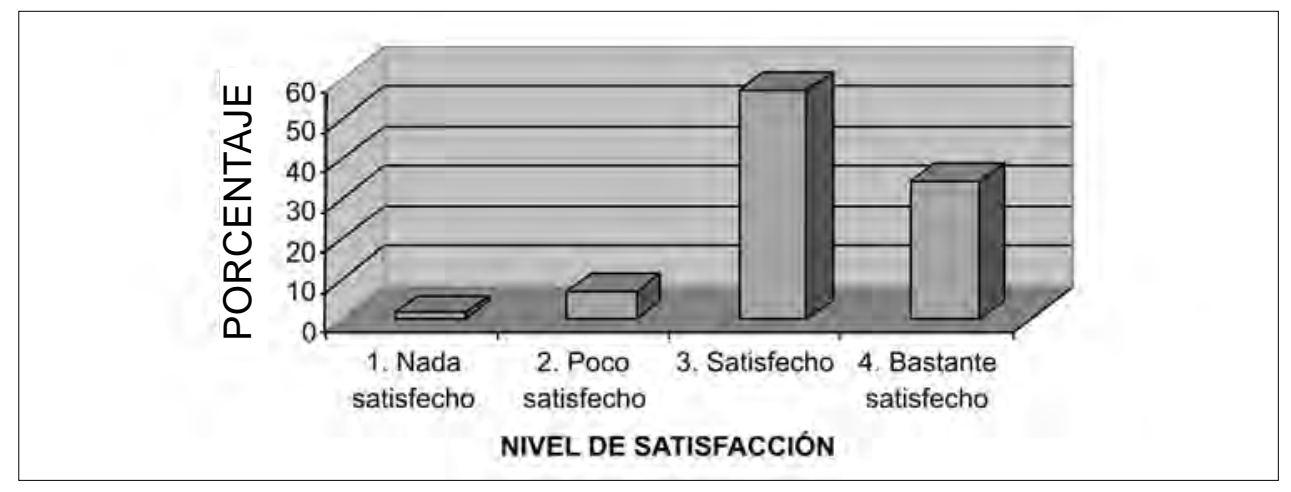

Gráfico 5. Dimensión 3.

La inmensa mayoría, más del 90\%, muestra un alto grado de satisfacción en la construcción de los mapas relacionales que se realizan en los centros educativos de Educación Secundaria. Tan sólo un 8,6\% muestra su insatisfacción en este aspecto. Algo que puede considerarse lógico sobre todo en las estructuras relacionales primarias propias de cualquier organización.

En general, en lo referente a mis relaciones académicas y personales con los alumnos, me siento:

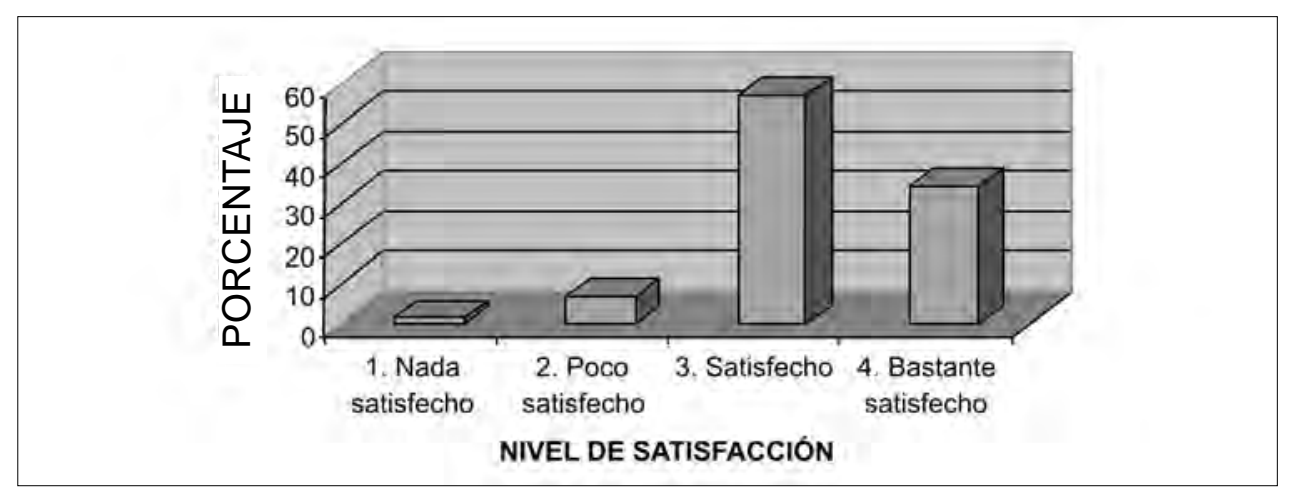

Gráfico 6. Dimensión 3. 
Igualmente aunque en menor porcentaje (88,5\%) se muestran satisfechos de las relaciones con los alumnos, tanto a nivel académico como personal. Es un dato importante en un contexto actual en el que se denotan actividades de violencia escolar e indisciplina en los centros educativos.

En general, con respecto a las relaciones con otros centros, me siento:

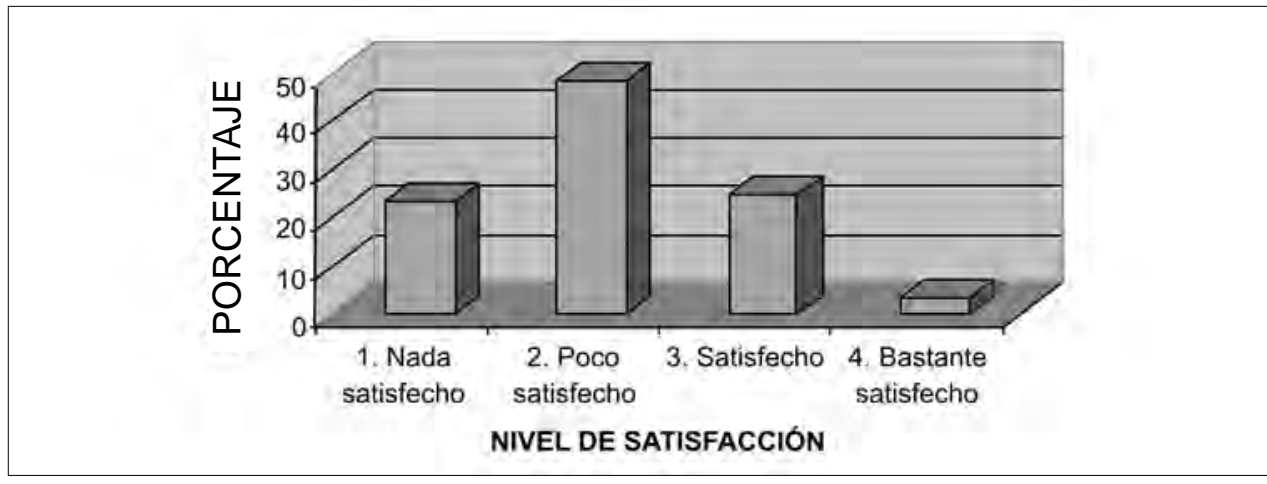

Gráfico 7. Dimensión 3.

Es muy alto el nivel de insatisfacción en cuanto a las relaciones con otros centros. El 62\% considera que es poco o nada satisfactorio, quizás por la cultura de balcanización y celularismo propia de nuestros centros educativos y quizás porque no Ilegue a existir tal relación. El trabajo de colaboración intercentros se muestra aquí muy escaso. El trabajo que desarrolla un profesor se limita en la mayor parte de los casos a la clase de sus alumnos y a la sala de profesores. A diferencia de otros sectores educativos la especialización de las áreas impartidas en educación secundaria hacen difícil las salidas a sectores relacionados con la misma.

\section{Dimensión 4. Nivel de insatisfacción-satisfacción con la estructura organizativa de los centros}

En general, con relación a la estructura organizativa del centro, me siento:

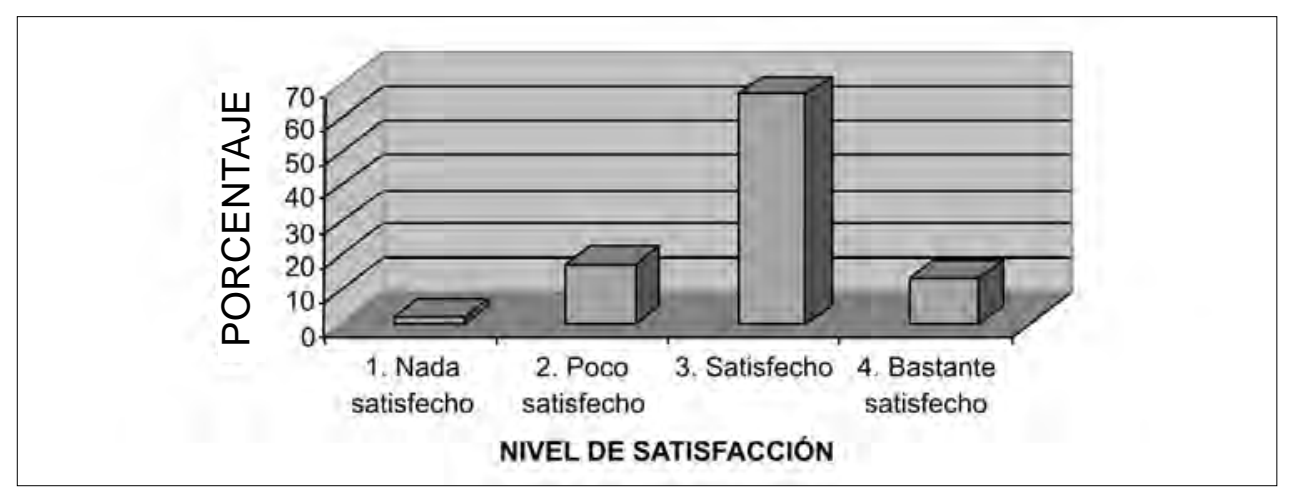

Gráfico 8. Dimensión 4. 
La estructura organizativa del centro referida a departamentos, procesos de evaluación, gestión de recursos, estructura de espacios-tiempos y horarios... satisface al profesorado en un alto porcentaje (80\%). Es interesante destacar que ciertamente hay satisfacción, pero el grado de esta satisfacción no es correlativo a la existencia del mismo.

\section{Dimensión 5. Nivel de insatisfacción-satisfacción con la estructura de la educación secundaria en el sistema educativo actual}

En general, con la estructura de la Educación Secundaria, me siento:

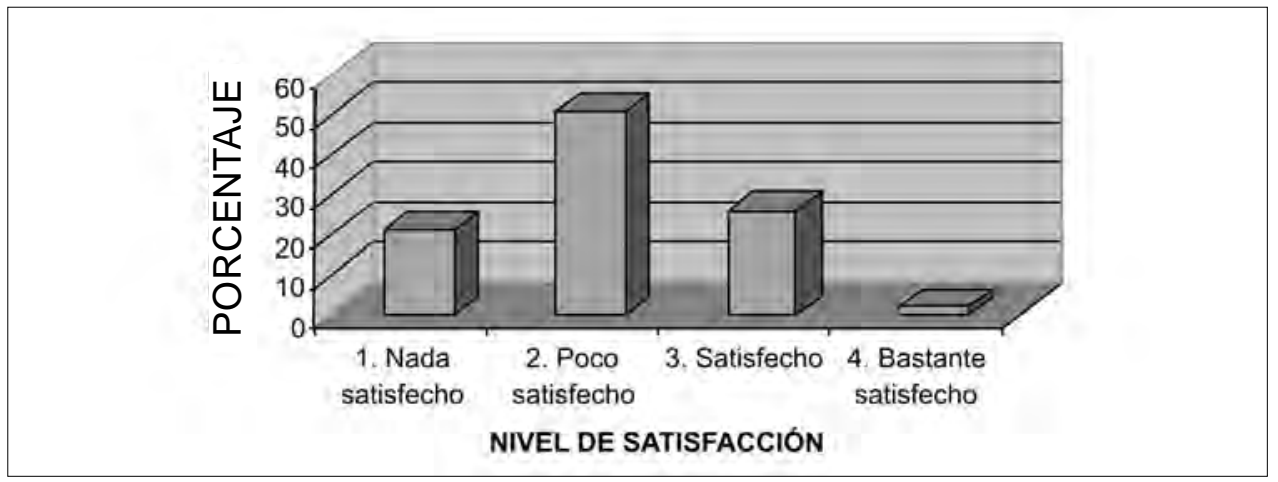

Gráfico 9. Dimensión 5.

A pesar de los diferentes cambios y reformas sufridas en nuestro país en los últimos años, parece que no se encuentra una estructura de esta etapa que satisfaga a los profesionales. Más del $70 \%$ no la encuentra adecuada y le genera insatisfacción sobre todo en cuanto a las etapas y la duración de las mismas.

\section{Dimensión 6. Nivel de insatisfacción-satisfacción con las bases teóricas que fundamentan el desarrollo normativo y organizativo de la educación secundaria}

En general, con las bases teóricas que fundamentan el sistema educativo actual, me siento:

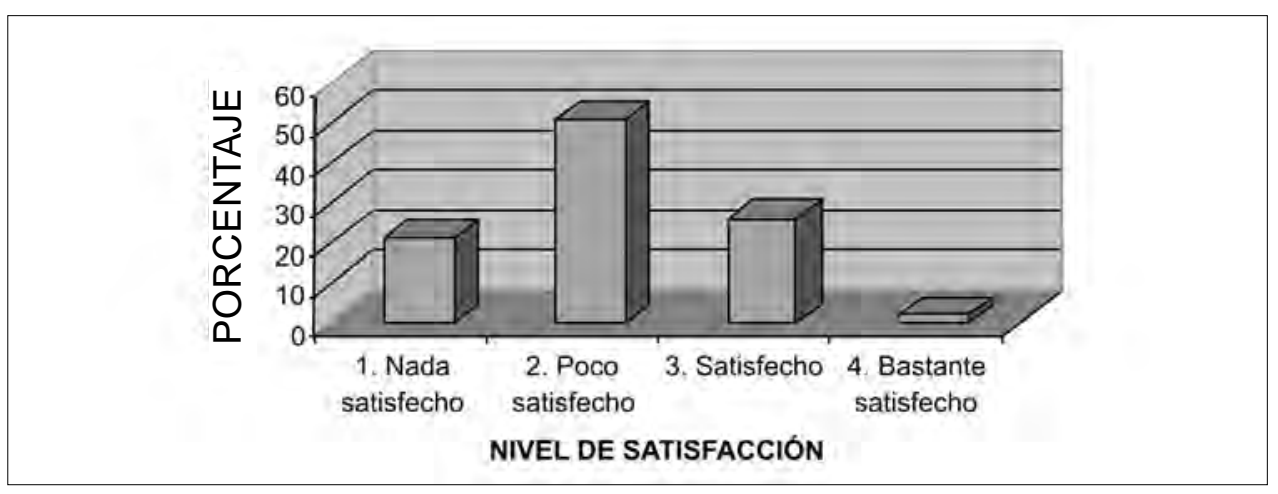

Gráfico 10. Dimensión 6. 
La mitad de los profesionales piensan que las bases teóricas (constructivismo, aprendizaje por descubrimiento...) no les satisfacen y optarían por unas bases más centradas en períodos anteriores (conductismo). Sin embargo los cambios generacionales indican un porcentaje muy parecido de profesionales que sí les satisfacen.

\section{ConClusiones}

Las primeras conclusiones que emanan de este trabajo se han podido observar claramente en el análisis de resultados. No obstante, queremos sintetizar las ideas más relevantes para posteriormente concluir las tendencias que se vislumbran en la investigación.

De manera general, el profesorado de Educación Secundaria se encuentra bastante satisfecho con el desarrollo de su profesión docente. El grado de satisfacción que ha exhibido en la mayor parte de los indicadores del cuestionario nos permite deducir que, a pesar de las dificultades, siguen trabajando, impartiendo sus clases con un grado de aceptación digno para sus alumnos y alcanzado cotas altas de participación e interrelación personal, tanto a nivel académico como personal, con sus alumnos y compañeros. Sin embargo, el grado de insatisfacción del profesorado se hace patente en la estructura relacional con la Administración Educativa y con las bases teóricas que sustentan el actual sistema educativo. En éste sentido, cuando el nivel de implicación no encuentra la satisfacción a las expectativas, al éxito en la acción, a la valoración de las cualidades y competencias y a la revalorización del status social, entre otros factores a considerar, los problemas pueden empezar a surgir.

El grupo de investigación analizará con posterioridad cada una de las dimensiones del cuestionario y tratará de cruzar las distintas variables que componen cada una de ellas. En ese trabajo estamos en la actualidad.

En resumen las tendencias que se observan son las siguientes:

1. La docencia genera entre la gran mayoría de los profesionales un gran nivel de satisfacción.

2. Ello se traduce en que ese mismo grado de satisfacción se extiende al ejercicio profesional que desarrollan. Disfrutan con su trabajo, creen que realizan una labor social útil. Entienden que contribuye a su realización personal por la libertad y el carácter humanístico de su tarea.

3. Entienden que el grado de aceptación que tienen con sus alumnos es elevado, al igual que las relaciones con sus compañeros. Acciones como la motivación de los alumnos, la ayuda a través de la acción tutorial, las estrategias para resolver conflictos, así como el respeto de los alumnos, les genera un alto grado de satisfacción. De igual manera, las relaciones con los colegas son satisfactorias en cuanto al trabajo cooperativo, las ayudas entre ellos y la confluencia de colegas de educación primaria en el centro, entre otras. Sin embargo, las relaciones con las familias, el grado de participación y la colaboración con profesores, les genera una fuerte insatisfacción, al igual que las relaciones con colegas de otros centros y con estos mismos a la hora de obtener información o compartir recursos. Este desánimo se manifiesta igualmente en las 
relaciones con la supervisión educativa y los equipos de orientación educativa, que entienden como algo adyacente a los centros.

4. En general, la estructura organizativa del centro les produce satisfacción. Sin embargo, no a todos los niveles: la ubicación de los centros en contextos desfavorecidos, la procedencia de los alumnos y las infraestructuras básicas les produce insatisfacción en su tarea. Son altos los porcentajes de satisfacción en cuanto a aspectos relacionados con la programación didáctica, la secuenciación de contenidos y los procesos de evaluación, así como sobre la estructura de los departamentos y sus niveles de coordinación.

5. La división de las etapas educativas en Educación Obligatoria, Bachillerato y Formación Profesional les genera un alto grado de insatisfacción. El mismo signo toma el tratamiento que se les da a las diferentes áreas curriculares en la ESO y los criterios de promoción de un ciclo a otro.

6. Por último, se muestran satisfechos con la autonomía de los centros y no con el grado de participación de la comunidad educativa. El profesorado está dividido en cuanto a las bases teóricas que sustentan la etapa, las bases pedagógicas, psicológicas y sociológicas del currículum. Más de la mitad no ha asumido estos principios y chocan en ocasiones con las escalas de valores que imperan en la sociedad actual.

Con todo lo expuesto podemos desmitificar algunas afirmaciones que se han dado por válidas hoy en día:

1. "El profesorado está insatisfecho". Demasiado simplista, ya que en realidad, los factores que influyen en esta insatisfacción son las relaciones con la administración educativa, con los padres de sus alumnos y con algunos compañeros. También se ve que la insatisfacción abarca hasta las relaciones con otros centros y a los cambios legislativos y teóricos del sistema educativo, ya que la estructura del actual sistema parece no conseguir los principales objetivos. Aunque, por el contrario, la estructura organizativa del centro suele compensar el trabajo de los docentes.

2. "El profesorado no se interesa por su alumnado". Sino todo lo contrario, las mayores satisfacciones se consiguen gracias a la relación que se produce alumno-profesor. No solo a nivel académico, sino también a nivel personal.

Podemos, para concluir, definir un docente de educación secundaria como una persona satisfecha con la docencia y el ejercicio de su profesión; que mantiene un grado de poca satisfacción con respecto a los supervisores educativos; que mantiene una relación delicada en relación a la familia; que con sus compañeros está satisfecho; y que en definitiva no comparte la estructura de la educación secundaria en la que desarrolla su trabajo pero que sí comparte las bases teóricas que la sustentan. Entendemos, por tanto, que el profesorado con un alto nivel de satisfacción en su tarea docente tiene actitudes positivas hacia el mismo; un profesional que se sienta insatisfecho tiene actitudes negativas hacia él. En este sentido se ha detectado que los factores más importantes que conducen a la satisfacción en el desempeño de la labor docente son: un trabajo desafiante desde el punto de vista mental, recompensas equitativas, condiciones de trabajo que constituyen un respaldo, colegas que 
apoyen y el ajuste personalidad-puesto. Entendemos que, desde el punto de vista de los profesionales, será bastante útil conocer las percepciones que tiene el profesorado sobre su nivel de satisfacción en su desarrollo profesional, sobre todo de cara a construir una identidad propia y a llevar a cabo proyectos de innovación que incidan en una mejora de la calidad de la educación.

\section{REFERENCIAS BIBLIOGRÁFICAS}

BOGLER, R. (2001). The influence of Leadership Style on Teacher Job Satisfaction. Educational Administration Quarterly, 5, 662-683.

CONLEY, S.; BACHARD, S. y BAUER, S. (1989). The school work environment and teacher career dissatisfaction. Educational Administration Quarterly, 25, 58-81.

DINHAM, S. y SCOTT, C. (2000). Moving into the third, outer domain of teacher satisfaction. Journal of Educational Administration, 4, 379-396.

ESTEVE, J. M. (1994). El malestar docente. Barcelona: Paidós Iberia S.A.

ESTEVE, J. M. (1998). El estrés de los profesores: Propuesta de intervención para su control. En A. Vila (coord.), Perspectivas y problemas de la función docente (pp.292-338). Madrid: Narcea.

EVANS, L. (2000). The effects educational change on morale, job satisfaction and motivation. Journal of educational Change, 1, 173-192.

GONZÁlEZ RíO, M. J. (1997). Metodología de la Investigación Social. Técnicas de recolección de datos. Alicante: Aguaclara.

GONZÁLEZ BLASCO, P. y GONZÁLEZ ANLEO, J. (1993). El profesorado en la España actual. Madrid: SM.

GORDILLO, V. (1988). La satisfacción profesional del profesorado: Consecuencias para la orientación educativa. En A. Villa. (coord.), II Congreso Mundial Vasco. Perspectivas y problemas de la función docente (pp. 259-266). Madrid: Narcea.

INCE (2003): Evaluación de la Educación Secundaria Obligatoria, 2000: informe final. Madrid, MECD.

MARCELO, C. (1992). Aprender a enseñar. Un estudio sobre el proceso de socialización de los profesores principiantes. Madrid: MEC. CIDE.

MARCELO, C. (1995). Formación del profesorado para el cambio educativo. Barcelona: PPU.

MARCHESI, A. (1990). Profesores, Centros docentes y calidad de la educación. Cuadernos de pedagogía, 184, 10-14.

MARCHESI, A. (2003): Indicadores de la educación en España y cambio educativo, en Revista de Educación, 330, pp.13-34.

MARCHESI, A. y MARTÍN, E. (2002): Evaluación de la Educación Secundaria. Fotografía de una etapa polémica. Madrid, Fundación Santa María.

MARTÍN, E.; PÉREZ, E. y ÁLVAREZ, N. (2007). La opinión del profesorado sobre la calidad de la educación. Madrid. FUHEM.

ORTIZ, V. (1993). El malestar del docente: la enseñanza media como unidad de referencia empírica. Universidad de Salamanca. Tesis Doctoral. 
PADRÓN, M. (1994). Satisfacción profesional del profesorado. Universidad de La Laguna. Tesis Doctoral.

PEIRÓ, J. M.; LUQUE, O.; MELIÁ, J. y LOSCERTALES, F. (1991). El estrés de enseñar. Sevilla: Alfar.

PÉREZ, A. I.; SOLA, M.; BLANCO, N. y BARQUÍN, J. (2003): Luces y sombras en la situación profesional de los docentes españoles». En J. Gimeno y J. Carbonell: El sistema educativo. Una mirada crítica. Madrid: Praxis Psicología.

PÉREZ, R. (1997). La profesión de docente. Estudio de las condiciones para el desarrollo de los profesores. Oviedo: Servicio de publicaciones de la Universidad.

SALANOVA, M. (2003). Burnout en profesores de enseñanza secundaria: un estudio longitudinal. En I. Martínez, M. Salanova, S. Llorens, M. García, R. Grau, y E. Cifre (Eds.), La enseñanza y el burnout: ¿una simbiosis irreversible? Castellón: Colección Psyque.

SEVILLA, U. y VILLANUEVA, R. (2000). La Salud Laboral Docente en la Enseñanza Pública. Madrid: Publicaciones de la Federación de Enseñanza de CCOO.

WALKER, J. C. y EVERS, C. W. (1988). The Epistemological Unity of Educational Research. En J.P. Keeves (Ed.): Educational Research Methodology, and Measurement: an International Handbook. Oxford: Pergamon Press.

VEENMAN, S. (1988). El proceso de llegar a ser profesor: un análisis de la formación inicial. En A. Villa. (coord.), Perspectivas y problemas de la función docente. II Congreso Mundial Vasco (pp.39-68). Madrid: Narcea.

VERA, J. (1986) Incidencias de los factores socio-laborales en el profesor debutante. Universidad de Málaga. Tesis Doctoral. S/P.

VILLAR, F. DEL (1993). El desarrollo del conocimiento práctico de los profesores de Educación Física, a través de un programa de análisis de la práctica docente. Un estudio de casos en formación inicial. Universidad de Granada. Tesis Doctoral inédita.

ZUBIETA, J. C. y SUSINOS, T. (1992). Las satisfacciones e insatisfacciones de los enseñantes. Madrid: CIDE. 